\title{
Atrial-specific pathways for control of intracellular signaling and myocyte function
}

\author{
Thomas J. Hund ${ }^{1,2,3}$ and Peter J. Mohler ${ }^{1,3,4}$ \\ 'Dorothy M. Davis Heart and Lung Research Institute, ${ }^{2}$ Department of Biomedical Engineering, ${ }^{3}$ Department of Physiology and Cell Biology, and ${ }^{4}$ Department of Internal Medicine, The Ohio State University \\ Wexner Medical Center, College of Medicine, and The Ohio State University College of Engineering, Columbus, Ohio, USA.
}

\begin{abstract}
Atrial fibrillation (AF) is a cardiac arrhythmia that arises from electrical and contractile dysfunction in the atria. Atrial function is regulated by a variety of intracellular signaling networks that facilitate rapid communication and coordinate responses of atrial myocytes. In this issue of the $J C l$, Brandenburg and colleagues describe the identification and characterization of "superhub" signaling nodes located on atrial axial tubules that regulate atrial contraction. Together, the results of this study provide important insight into the regulation of atrial contraction and describe potential therapeutic targets to be explored in future studies.
\end{abstract}

\section{Intricate regulatory networks} determine atrial function

Atrial fibrillation (AF) is the most common sustained arrhythmia, affecting more than three million people in the United States $(1,2)$. While specific defects in atrial myocyte excitability and structure have been associated with $\mathrm{AF}$ in a variety of cardiac disease states (or even in normal aging), our understanding of pathways for regulation of atrial myocyte excitability in normal and diseased conditions is incomplete (3). Given the prevalence of atrial arrhythmias and the limitations of existing therapies including off-target effects, procedural complications, and poor efficacy, there clearly remains a need for a deeper understanding of atrial myocyte excitability and the regulatory pathways involved, with the hope of identifying potential therapeutic targets (4).

Cardiac myocytes have evolved an elaborate web of intracellular signaling networks to rapidly tune cell (and ultimately organ) function in response to stress. While these signaling pathways show tremendous variety with respect to constituent signaling molecules, upstream effectors, downstream targets, and emergent function, these networks have several commonalities, including a dependence on reversible posttranslational modification as a way to rapidly alter target protein activity and/or localization (5). Specialized myocyte membrane domains are critical for maintaining temporal and spatial control over intracellular signaling, and defects in local signaling are commonly linked to decreased function and arrhythmia in cardiac disease. Among the best characterized of these myocyte membrane domains are transverse tubule (TT) membranes, sarcolemmal membrane invaginations that occur near Z-lines and support synchronous myocyte contraction (Figure 1). TT membranes are home to a variety of important signaling domains, most notably the cardiac dyad formed by close apposition of L-type $\mathrm{Ca}^{2+}$ channels in the TT membrane and RyR2 sarcoplasmic reticulum (SR) $\mathrm{Ca}^{2+}$ release channels. RyR2 channels themselves interact with a large number of accessory, adapter, and regulatory proteins to form unique local signaling domains. The adapter protein ankyrin-B (AnkB) also localizes to the TT membrane and coordinates the organi-

\section{Related Article: p. 3999}

zation of distinct macromolecular complexes involving, among other proteins, the inositol 1,4,5 trisphosphate (IP3) receptor and protein phosphatase $2 \mathrm{~A}$ (PP2A) (5). Defects in AnkB have been linked to abnormal $\mathrm{Ca}^{2+}$ homeostasis and membrane excitability, as well as both ventricular and atrial arrhythmias (5).

\section{A "super-hub" of signaling} Compared with those in ventricular myocytes, TTs in atrial myocytes are relatively underdeveloped; however, atrial cells have evolved unique membrane domains to efficiently regulate myocyte function, reflecting their unique structure and energetic demands. In this issue, Brandenburg and colleagues use a host of innovative imaging modalities and markedly expand our understanding of local signaling domains in atrial myocytes (6). Brandenburg et al. propose a new model for atrial signaling based on the concept of local "superhub" signaling nodes located on atrial axial tubules (ATs). While ATs have been previously described in atria (7-9), this new work proposes that these domains display unique structural, signaling, and regulatory signatures that underlie atrial-specific regulation at baseline and in disease. Specifically, Brandenburg and colleagues report the identification of atrial AT "super-hub" domains that are unusually large and robust. In fact, AT domains were found to be more abundant in atria than in ventricles, more prevalent than TTs in the atria, and to display increased width and volume/surface area ratios compared with the atrial TTs that connect the AT domains. Second, in line with previous findings (10), these atrial AT "hubs" were shown to harbor functional dyads, including L-type calcium channels (Cav1.2) and ryanodine receptor 2 (RyR2) calcium-release channels, as well as caveolin 3 (Cav3), an integral component of the caveolae membrane. 
A

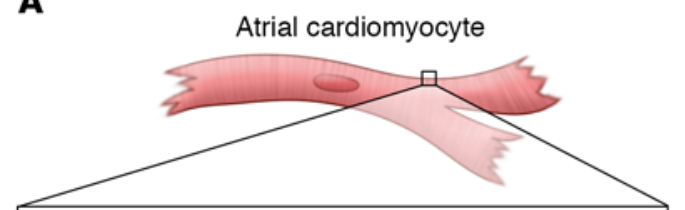

Extracellular space

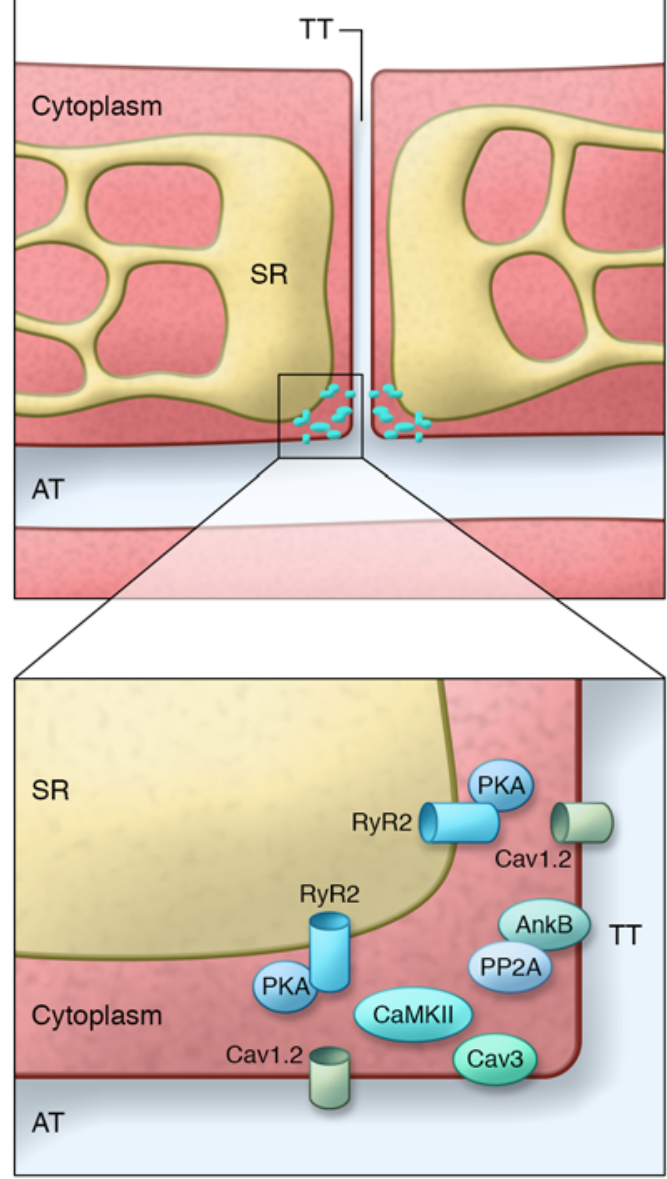

B
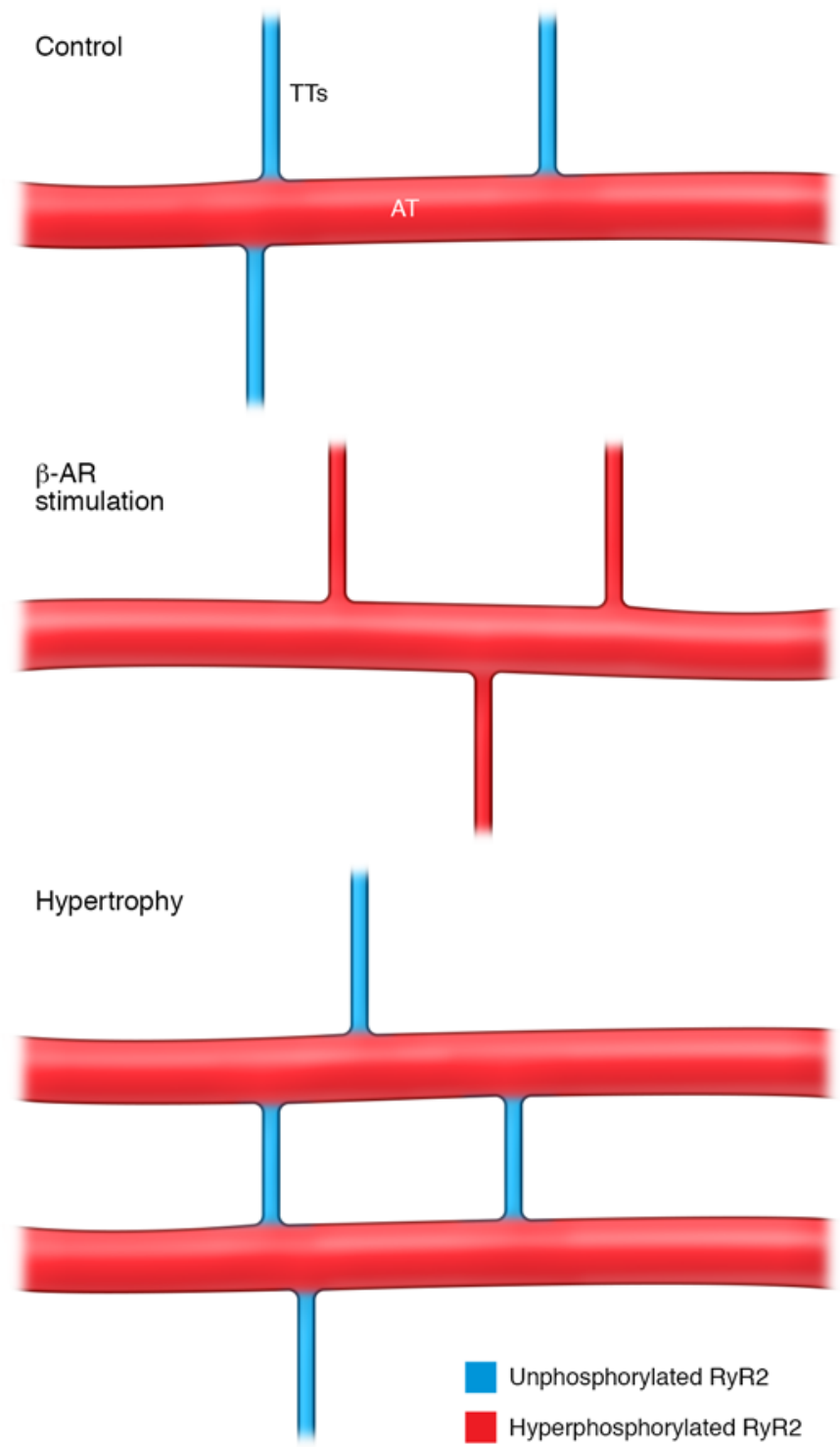

Figure 1. Cardiac atrial signaling "hubs" regulate local calcium signaling. (A) Schematic of the atrial myocyte TT and AT networks and the SR. In this issue, Brandenburg et al. have described an atrial "super hub" (inset), which harbors a unique signaling platform with CRUs and regulatory proteins. RyR2 clusters are differentially regulated at TT and AT domains, potentially due to a distinct constituency of regulatory macromolecular complexes. (B) Schematic showing the distribution of hyperphosphorylated (red) and unphosphorylated (blue) CRUs in association with ATs and TTs at baseline, following $\beta$-AR stimulation, and in atrial hypertrophy.

Third and most notable, Brandenburg and et al. report that atrial AT RyR2 populations display unique regulatory properties. For example, canonical SR RyR2 populations juxtaposed with the peripheral sarcolemma were minimally phosphorylated under baseline conditions (in the absence of $\beta$-adrenergic receptor $[\beta-A R]$ stimulation), whereas "superhub" RyR2 calcium-release units (CRUs) were hyperphosphorylated at both protein kinase A-dependent (PKA-dependent) and $\mathrm{Ca}^{2+}$ calmodulin-dependent protein kinase II-dependent (CaMKII-dependent) phosphorylation sites. Brandenburg et al. propose that hyperphosphorylation of AT RyR2 increases open probability $\left(\mathrm{P}_{\mathrm{o}}\right)$, particularly in situations with an elevated SR $\mathrm{Ca}^{2+}$ load, and supports rapid $\mathrm{Ca}^{2+}$-release events that precede canonical subsarcolemmal $\mathrm{Ca}^{2+}$ release. Further, the authors assert that this chamber-specific signaling pathway, at least in part, regulates rapid activation of atrial myofilaments and contractility, particularly in pathological situations (e.g., atrial hypertrophy). Finally, these studies suggest that this local "superhub" model may be ripe for targeting in future atrial therapies due to its unique structure and regulatory properties.

\section{Conclusions and future directions}

The study by Brandenburg and colleagues (6) is an experimental tour de force that tackles a fundamental yet understudied area of cardiovascular biology. By integrating the power of tried-and-true tech- 
nologies, such as transmission electron microscopy, with cutting-edge approaches, like super-resolution light microscopy, this research directly addresses highly important questions regarding the links between atrial structure and signaling, function, and subsequent downstream cardiovascular phenotypes. Importantly, this study by Brandenburg et al. adds to a wealth of data in the field supporting the notion that cell size is not the only differentiating factor in discriminating atrial from ventricular cells. Certainly, we now know that, in addition to unique membrane morphologies, the signature of ion channels, transporters, signaling molecules, and transcriptional regulatory programs that regulate atrial excitation-contraction coupling is distinct $(11,12)$. With the advent of new high-resolution imaging technologies and more sensitive proteomic approaches, we will likely soon witness a host of data that will define new chamber-specific pathways that may aid the design of more sensitive disease diagnostics and effective therapeutics.

As with any study that touches so many areas of biology, there are a host of future directions that will be critical for the field to address. First, how has the local environment of the atrial AT evolved to favor hyperphosphorylation of CRUs in the absence of $\beta$-AR stimulation? Certainly, increased basal cAMP and/or reduced basal phosphodiesterase activity would push this balance toward RyR2 hyperphosphorylation. Alternatively, a reduction in local phosphatase activities (protein phosphatases PP2A and PP1) would support this model, potentially due to distinct targeting/regulatory subunit expression (13-15). In this regard, it is interesting to consider the possibility that a differential phosphorylation status in atrial RyR2 populations may stem from differential expression of adapter/scaffolding proteins, such as AnkB, that are important for the control of local signaling, rather than from the signaling molecules themselves. Second, relevant to the data presented by Brandenburg et al., RyR2 hyperphosphorylation is tightly linked with altered SR calcium release and AF (16); therefore, how has this local "super-hub" environment evolved to mimic a height- ened adrenergic status of RyR2 without becoming arrhythmogenic? Third, what is the relationship of the axial TT with the canonical TT in atria or ventricles? Is this membrane structure biochemically unique with regard to lipid and protein organization? Further, are there unique components of SR that are adjacent to this membrane structure? It will be important, going forward, to further characterize the distinct molecular constituents of TT and AT membranes to: (a) better understand the mechanism(s) underlying differential regulation of SR $\mathrm{Ca}^{2+}$ release; and (b) identify novel therapeutic targets for the selective control of atrial excitability. Fourth, are there other atrial ion channels in this "super-hub" that alter local atrial signaling? For example, it is interesting to consider that a subpopulation of $\mathrm{Na}_{\mathrm{v}} 1.5$ channels that are distinct from those that are a major component at the intercalated disc may regulate local signaling at the AT. This possibility is especially intriguing in light of recent data showing that CaMKII-dependent regulation of $\mathrm{Na}_{\mathrm{v}} 1.5$ is involved in the pathophysiological response to local adrenergic signaling (17-19). Finally, how do the atrial AT network and the "super-hub" remodel in response to both physiological and pathological stresses? While the study by Brandenburg and colleagues supports the idea that the AT network proliferates following atrial hypertrophy (6), prior studies indicate that transverse AT networks are lost in large animal models of persistent $\operatorname{AF}(7,20)$. It will be critical in the future to extend these studies to continue to push our fundamental understanding of atrial biology to aid in our discovery of new therapies for common forms of AF.

\section{Acknowledgments}

The authors are supported by grants from the NIH (HL114893, to TJH and HL084583, HL083422, and HL114383, to PJM); the James S. McDonnell Foundation (to TJH); the American Heart Association; and the William D. and Jacquelyn L. Wells Fund for Cardiovascular Research.

Address correspondence to: Peter J. Mohler, The Ohio State University Wexner Medical Center, 473 W. 12th Avenue,
DHLRI 110G, Columbus, Ohio 43210, USA. Phone: 614.292.5019; E-mail: Peter. mohler@osumc.edu.

1. Naccarelli GV, Varker H, Lin J, Schulman KL. Increasing prevalence of atrial fibrillation and flutter in the United States. Am J Cardiol. 2009;104(11):1534-1539.

2. Go AS, et al. Executive summary: heart disease and stroke statistics - 2014 update: a report from the American Heart Association. Circulation. 2014;129(3):399-410.

3. Heijman J, Voigt N, Nattel S, Dobrev D. Cellular and molecular electrophysiology of atrial fibrillation initiation, maintenance, and progression. Circ Res. 2014;114(9):1483-1499.

4. Hohnloser SH, Singh BN. Proarrhythmia with class III antiarrhythmic drugs: definition, electrophysiologic mechanisms, incidence, predisposing factors, and clinical implications. J Cardiovasc Electrophysiol. 1995; 6(10 pt 2):920-936

5. Curran J, Mohler PJ. Alternative paradigms for ion channelopathies: disorders of ion channel membrane trafficking and posttranslational modification. Annu Rev Physiol. 2015; 77:505-524

6. Brandenburg S, et al. Axial tubule junctions control rapid calcium signaling in atria. J Clin Invest. 2016;126(10):3999-4015.

7. Dibb KM, et al. Characterization of an extensive transverse tubular network in sheep atrial myocytes and its depletion in heart failure. Circ Heart Fail. 2009;2(5):482-489.

8. Frisk M, Koivumäki JT, Norseng PA, Maleckar MM, Sejersted OM, Louch WE. Variable t-tubule organization and $\mathrm{Ca}^{2+}$ homeostasis across the atria. Am J Physiol Heart Circ Physiol. 2014;307(4):H609-H620.

9. Glukhov AV, et al. Direct evidence for microdomain-specific localization and remodeling of functional L-type calcium channels in rat and human atrial myocytes. Circulation. 2015;132(25):2372-2384.

10. Kirk MM, et al. Role of the transverse-axial tubule system in generating calcium sparks and calcium transients in rat atrial myocytes. JPhysiol (Lond). 2003;547(pt 2):441-451.

11. Weckbach LT, Grabmaier U, Clauss S, Wakili R. MicroRNAs as a diagnostic tool for heart failure and atrial fibrillation. Curr Opin Pharmacol. 2016;27:24-30.

12. Voigt N, Dobrev D. Atrial-Selective Potassium Channel Blockers. Card Electrophysiol Clin. 2016;8(2):411-421.

13. DeGrande ST, et al. Molecular mechanisms underlying cardiac protein phosphatase $2 \mathrm{~A}$ regulation in heart. $\mathrm{JBiol}$ Chem. 2013;288(2):1032-1046.

14. Little SC, et al. Protein phosphatase $2 \mathrm{~A}$ regulatory subunit B56 $\alpha$ limits phosphatase activity in the heart. Sci Signal. 2015;8(386):ra72.

15. Chiang DY, et al. Impaired local regulation of ryanodine receptor type 2 by protein phosphatase 1 promotes atrial fibrillation. Cardiovasc Res. 2014;103(1):178-187.

16. Chelu MG, et al. Calmodulin kinase II- 
mediated sarcoplasmic reticulum $\mathrm{Ca}^{2+}$ leak promotes atrial fibrillation in mice. JClin Invest. 2009;119(7):1940-1951.

17. Glynn P, et al. Voltage-gated sodium channel phosphorylation at Ser571 regulates late current, arrhythmia, and cardiac function in vivo. Circulation. 2015;132(7):567-577.
18. Koval OM, et al. $\mathrm{Ca}^{2+} /$ calmodulin-dependent protein kinase II-based regulation of voltage-gated $\mathrm{Na}^{+}$channel in cardiac disease. Circulation. 2012;126(17):2084-2094.

19. Hund TJ, et al. A $\beta(I V)$-spectrin/CaMKII signaling complex is essential for membrane excitability in mice. JClin Invest.
2010;120(10):3508-3519.

20. Lenaerts I, et al. Ultrastructural and functional remodeling of the coupling between $\mathrm{Ca}^{2+}$ influx and sarcoplasmic reticulum $\mathrm{Ca}^{2+}$ release in right atrial myocytes from experimental persistent atrial fibrillation. Circ Res. 2009;105(9):876-885. 